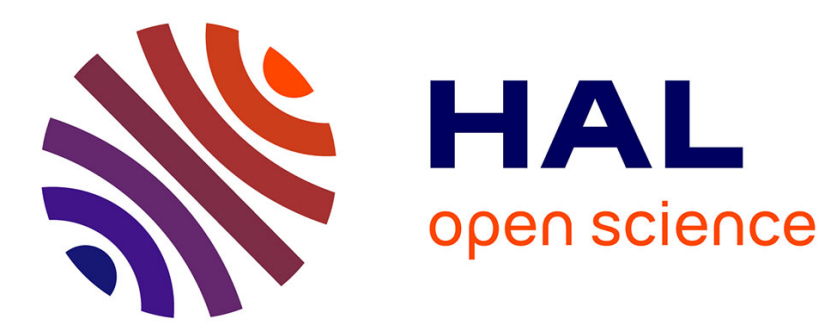

\title{
2D nanostructure motion on anisotropic surfaces controlled by electromigration
}

Stefano Curiotto, Pierre Müller, Ali El-Barraj, Fabien Cheynis, Olivier Pierre-Louis, Frédéric Leroy

\section{- To cite this version:}

Stefano Curiotto, Pierre Müller, Ali El-Barraj, Fabien Cheynis, Olivier Pierre-Louis, et al.. 2D nanostructure motion on anisotropic surfaces controlled by electromigration. Applied Surface Science, 2019, 469 (1), pp.463-470. 10.1016/j.apsusc.2018.11.049 . hal-01889868v2

\section{HAL Id: hal-01889868 \\ https://hal.science/hal-01889868v2}

Submitted on 14 Nov 2018

HAL is a multi-disciplinary open access archive for the deposit and dissemination of scientific research documents, whether they are published or not. The documents may come from teaching and research institutions in France or abroad, or from public or private research centers.
L'archive ouverte pluridisciplinaire HAL, est destinée au dépôt et à la diffusion de documents scientifiques de niveau recherche, publiés ou non, émanant des établissements d'enseignement et de recherche français ou étrangers, des laboratoires publics ou privés. 
published on Applied Surface Science $469(2019) 463-470$
https://doi.org/10.1016/j.apsusc.2018.11.049

\title{
2D nanostructure motion on anisotropic surfaces controlled by electromigration
}

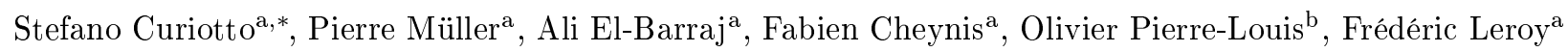 \\ ${ }^{a}$ Aix Marseille Univ, CNRS, CINAM, Marseille, France \\ ${ }^{b}$ Institut Lumière Matière, UMR 5306 Université Lyon 1-CNRS, Universitè de Lyon, 69622 Villeurbanne, France
}

\begin{abstract}
Nanostructures on surfaces can be displaced by applying an electric field or electric currents through a material. This induced mass transport is referred to as electromigration. In this article we show that the anisotropy of diffusion may control the direction of motion of electromigrating nanostructures. For this purpose we study in situ and in real time, by Low Energy Electron Microscopy, the motion of 2D one-atom thick islands or one-atom deep holes on a highly anisotropic surface (reconstructed $\mathrm{Si}(100)$ ). Based on experiments and Kinetic Monte Carlo simulations, we propose a simple analytical model that explains most of the observations. In particular, the direction of motion of the nanostructures depends on the diffusion anisotropy and does not necessarily coincide with the electric field direction. This work opens a way for the manipulation of $2 \mathrm{D}$ nanostructures by means of an electric field on anisotropic surfaces.
\end{abstract}

\section{Introduction}

The manipulation of nanostructures is an important challenge for nanoelectronics and nanofabrication technologies. External fields and thermal gradients are natural candidates to move and control the nanostructure position on a surface. Particularly promising is the use of electric fields to induce mass transport in specific directions, phenomenon known as electromigration [1]. This phenomenon has been widely studied in another context since the sixties: an electric current flowing through a circuit, if not controlled, can lead to the formation of hillocks and voids resulting in breakdown of metallic wires 2 . The elementary process of electromigration of an atom on a surface (adatom) is well known: the electric current biases random diffusion inducing mass transport in specific directions. The phenomenon is due to either kinetic momentum exchange between conducting electrons and adatoms (wind force) or because of electrostatic forces (direct force). The atoms are thus described as carrying an effective charge that results from the contribution of these two forces. The drift of adatoms along facets or terraces does not necessarily result in a motion of nanostructures. Indeed, if the number of atoms arriving and attaching to a step edge is equal to the number of atoms leaving the step, then that edge does not move. An imbalance between the two fluxes is needed to allow for the motion of the edge. This imbalance can for instance originate from an Ehrlich-Schwoebel

\footnotetext{
* Corresponding author

Email addresses: curiotto@cinam.univ-mrs.fr (Stefano Curiotto), muller@cinam. univ-mrs.fr (Pierre Müller), elbarraj@cinam.univ-mrs.fr (Ali El-Barraj), cheynis@cinam. univ-mrs.fr (Fabien Cheynis), olivier.pierre-louis@univ-lyon1.fr (Olivier Pierre-Louis), leroy@cinam.univ-mrs.fr (Frédéric Leroy)
}

barrier (an inequality of atomic attachment and detachment properties at steps [3, 4]) or a different diffusion coefficient at the two sides of the edge. While it has been neglected in most studies, the crystalline anisotropy of the surface may play an important role on adatom motion and therefore on nanostructure motion.

To investigate the motion of nanostructures under electromigration on anisotropic surfaces we have chosen $\mathrm{Si}(100)$ as a model system. The effect of electromigration on the pairing of steps and on step bunching in this system has been studied by several authors [5-11]. The anisotropy of the diffusion coefficient has been found to play an important role, however the effect of the coupling between the electric current direction and surface anisotropy on the motion of nanostructures (like one-atom thick islands or one-atom deep holes) still needs to be adressed.

The experimental conditions and instrumental tools required to fully characterize the motion of nanostructures on mesoscopic length are strongly constrained: (i) The material surfaces must be flat enough to avoid obstacles on the nanostructure trajectory; (ii) an in-situ and in real-time multiscale experimental technique is necessary to obtain simultaneous information on the kinetics of the nanostructure motion and on the shape evolution; (iii) a large temperature range must be explored in order to get information on the thermodynamic behavior of the moving nanostructures. Following the evolution of scientific equipments, first in-situ experimental studies on electromigration have used Reflection Electron Microscopy (REM), while most recent investigations are based on Scanning Tunneling Microscopy (STM). However both approaches have their own limit. In REM experiments, due to the glancing conditions for the incident electron beam, the images are severely shortened along one axis. Therefore the shape evolution of a moving nanostructure cannot be studied [12, 13. Con- 
ventional STM experiments only give access to the low temperature regime $(<500 \mathrm{~K})$ and do not allow to study fast moving and/or large nanostructures. In order to overcome these limitations we have used Low Energy Electron Microscopy (LEEM), that allows one to observe surfaces in-situ and in real time with high temporal and spatial resolution, and in a large temperature range (1000-1500 K). Furthermore, we have used lithographically prepared surfaces to obtain atomically flat terraces with micrometric sizes on which $2 \mathrm{D}$ nanostructures may be displaced on large distances without interactions with steps.

In order to support the experimental results and obtain more insights in the motion mechanism, we have developed a Kinetic Monte Carlo model based on both anisotropic and electromigration-biased diffusion at surfaces. Nanostructure motion biased by electromigration on anisotropic surfaces may be understood on the basis of simple schemes, which nevertheless may lead to unexpected motions. For instance, since electromigration affects mass transport, one could expect that a nanostructure moves along the current axis, either in the electric field direction or opposite to it, depending on the nature of the nanostructure $(2 \mathrm{D}$ hole or $2 \mathrm{D}$ island) and on the main mechanism at work (surface diffusion, periphery diffusion or attachment/detachment). Counter-intuitively we show that, due to the anisotropy of the surface diffusion coefficient, nanostructures can also move perpendicularly to the electron flow. We have derived a simple analytical expression to describe the motion of $2 \mathrm{D}$ nanostructures under an electric field in case of anisotropic diffusion. Furthermore, since working in a large temperature range, an effective activation energy for the motion of $2 \mathrm{D}$ nanostructures has been obtained. This energy is given by two contributions, a diffusion energy and an adatom formation energy.

\section{Experimental details}

Single-crystal B-doped (p-type) silicon wafers with 500 $\mu \mathrm{m}$ thickness, nominal orientation (100), and a miscut of $\pm 0.01^{\circ}$ were supplied by Siltronix. Circular holes $7.5 \mu \mathrm{m}$ deep, with $300 \mu \mathrm{m}$ diameter separated by $100 \mu \mathrm{m}$ were prepared by standard optical lithography using a hexagonal mask. The holes were made to interrupt the regular train of steps and allow formation of large terraces, as suggested by [14]. Then, the wafers were cut in laths $3 \mathrm{~mm}$ wide, $12 \mathrm{~mm}$ long, and cleaned with acetone and ethanol. According to the sample, the long side is aligned along the [011] or the [010] direction. They were mounted on a special sample-holder (designed by Elmitec Gmbh) which allows to apply an electric current through the sample by means of two Mo electrodes clamped to the extremities of the sample. The current direction was parallel to the longer side of the sample and the applied electric current was typically within the range $0.5-3 \mathrm{~A}$. The samples were introduced in Ultra High Vacuum (UHV) and degassed for several hours at about $1100 \mathrm{~K}$. Then they were flashed above $1500 \mathrm{~K}$ to remove the surface oxide and the first $\mathrm{Si}$ layers. The temperature was decreased and kept for some minutes at $1300 \mathrm{~K}$, where fast surface diffusion, slow $\mathrm{Si}$ evaporation and electromigration [6] allow to increase the size of the terraces up to some $\mu \mathrm{m}$. The temperatures were measured with a precision of $30 \mathrm{~K}$, using a disappearingfilament pyrometer, calibrated with the $7 \times 7$ to $1 \times 1$ surface phase transition occurring on $\operatorname{Si}(111)$ at $1103 \mathrm{~K}$ [15]. The in-situ observations were performed in a Low Energy Electron Microscope (LEEM III, Elmitec Gmbh), in dark field mode, using electron energies ranging between 4 and $6 \mathrm{eV}$. The images were acquired at rates of $0.1-1 \mathrm{~Hz}$. Single-step holes have been made by evaporation in large terraces, increasing the current through the sample and therefore increasing the temperature (above $1340 \mathrm{~K}$ ) and observing in real time by LEEM their formation. When the desired hole size is reached, the temperature is lowered to stop evaporation and the sample is kept at a certain temperature and observed to study the hole motion dynamics. Islands can also be created by depositing in-situ less than 1 mono-layer of $\mathrm{Si}$, up to observe the formation of the islands; their number and size can be tuned by changing the substrate temperature, the flux or the deposition time.

\section{Experimental results}

The reconstructed surface of $\mathrm{Si}(100)$ is arranged in parallel dimer rows running along the [011] or the [01-1] axis: the direction rotates by $90^{\circ}$ from one terrace to the next separated by a monoatomic step (see Figure 1 a). The terraces therefore give different diffraction spots in Low Energy Electron Diffraction (LEED). Thus in dark field LEEM, successive terraces appear alternatively dark and bright. Figure 1 b shows dark ellipses, that correspond to one-atom deep holes, in a $\mathrm{Si}(100)$ terrace. The long axis of the elliptic holes is oriented along the dimer rows of the upper terrace (schematically drawn with parallel lines in Figure 1). To obtain these one-atom deep holes on an extended terrace, the sample temperature is first raised to increase the evaporation rate and to nucleate and grow holes. Then the temperature is decreased to reach the desired working conditions (typical temperatures of 1080$1300 \mathrm{~K})$. We have changed the direction of the current with respect to the direction of the dimer rows and observed the motion of nanostructures, in order to study the combined effect of electromigration and anisotropic diffusion.

When an electric current is applied through the sample in the [011] direction, the black holes, elongated perpendicularly to the current, move opposite to the direction of the current, as shown in Figure 1 taken $278 \mathrm{~s}$ after 1b. Similarly, Figures $1 \mathrm{~d}$ and 1e show the motion of holes elongated in the current direction (dimer rows of the upper terrace parallel to the current). In this case the holes (bright because their dimer rows are perpendicular to the dimer rows of the holes in Figures $1 \mathrm{p}$ and 1 ; ) move in the same direction as the current.

We have also applied a current in the [010] direction, not aligned along the dimer rows, but at $45^{\circ}$ from them. 

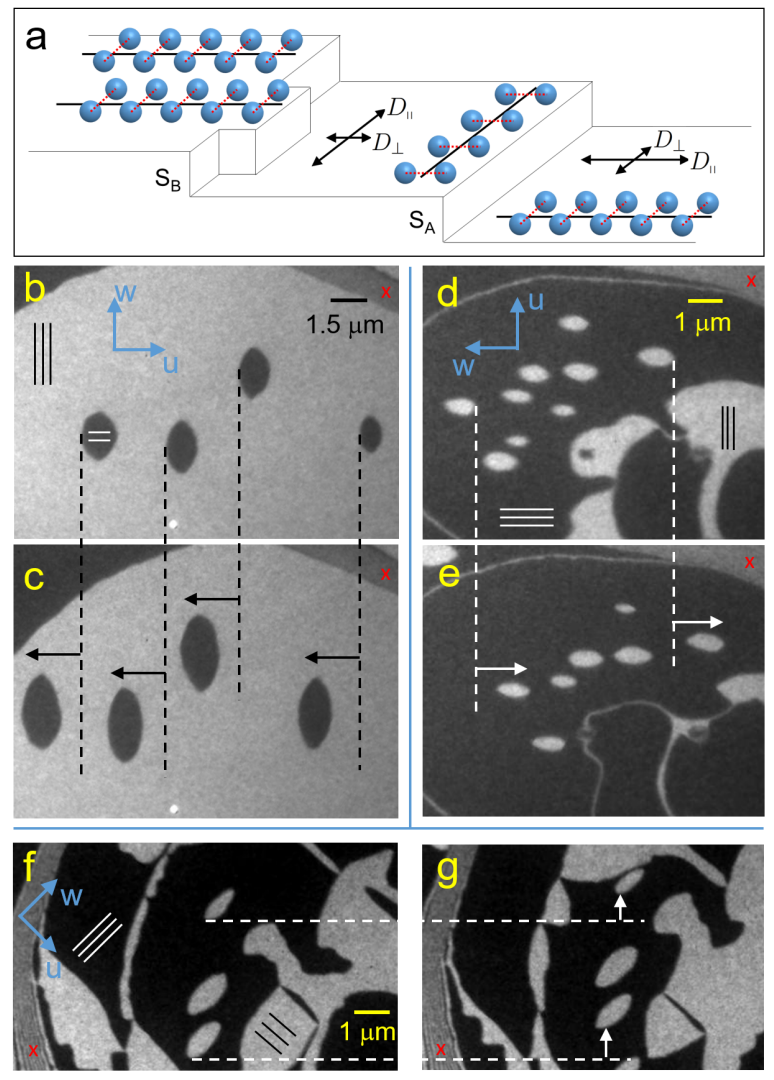

$\mathrm{F}$

Figure 1: a: Illustration of the $\mathrm{Si}(100)$ surface structure, with dimer rows running along the [011] or the [0-11] axis: the direction changes by $90^{\circ}$ in alternating terraces. $S_{B}$ steps, perpendicular to dimer rows, are rough, while $S_{A}$ steps, parallel to dimer rows, are flatter because of their higher step energy [16]. b-g: dark field LEEM images of one-atom deep holes in $\operatorname{Si}(100)(\mathrm{E}=4.5 \mathrm{eV})$. Under electromigration (current directed towards the right for all the images of the figure) the holes displace in different directions according to their orientation. $\vec{u}$ and $\vec{w}$ are shown to understand the model explained in section 4 and the schematics of Figure 2 The dimer rows inside the holes are oriented in the direction of $\vec{u}$, while outside the holes they are parallel to $\vec{w}$. Depending on the orientation of the dimer rows inside the holes, $\vec{u}$ and $\vec{w}$ are either in the [011] and [0-11] directions (b and c) or in the [0-11] and [0-1-1] directions (d and e). b-c: the holes with dimer rows running parallel to the force displace to the left, opposite to the electromigration force; d-e: the holes with dimer rows running perpendicularly to the force displace in the same direction of the force; f-g: when the holes have dimer rows at $45^{\circ}$ from the force direction, they displace perpendicularly to the force. The step bunches on the top right of $b, c, d$ and e and on the left of $f$ and $\mathrm{g}$ are the fixed reference regions (marked with a red cross). All the elliptical structures shown in this figure are holes but they are dark or bright according to the direction of their dimer rows and according to the diffraction spot used in the dark field image.

Figure 1f shows bright holes elongated diagonally, where the dimer rows of the upper dark terrace are oriented from the bottom left towards the top right of the image. Surprisingly, with a current applied as shown in the figure, from left to right, the holes move towards the top (Figure $1 \mathrm{~g})$, at $90^{\circ}$ from the electric-field direction and $135^{\circ}$ from the [011] axis.

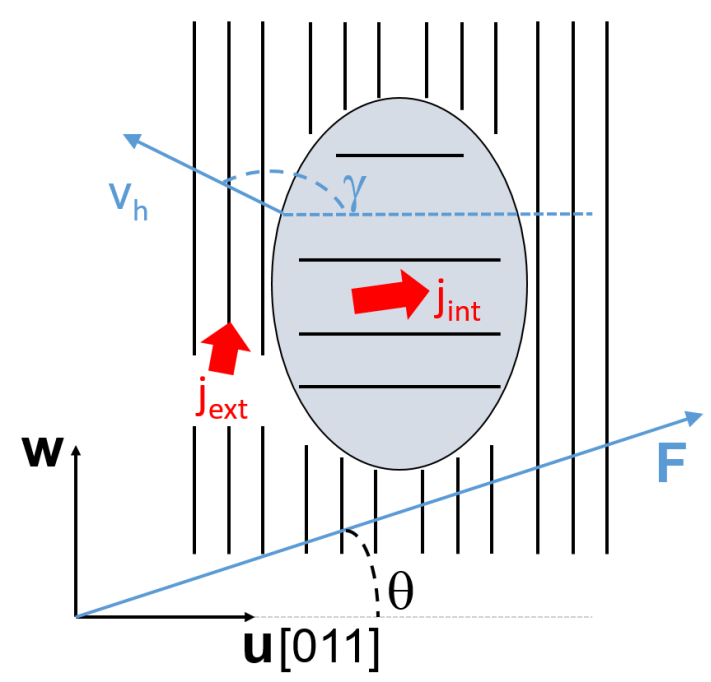

Figure 2: Schematics of a one-atom deep hole in $\mathrm{Si}(100)$. The dimer rows inside the hole are oriented horizontally, in the same direction of the $\vec{u}$ vector. The force exerted by the electric field is oriented along the blue arrow and forms an angle $\theta$ with the $\vec{u}$ vector. The hole displaces in a direction forming an angle $\gamma$ with $\vec{u}$.

We have also studied islands instead of holes. As shown in the movie of supplementary material S1, the islands displace opposite to the motion direction of holes with the same dimer row orientation.

The velocity of the motion does not depend on the size of holes or islands. Therefore according to established interpretations [17, the process of mass transport is limited by diffusion of adatoms on terraces and is not influenced by periphery diffusion or attachment detachment kinetics. For identical current-temperature conditions, the velocity of the nanostructure is independent from the orientation of the force (within the experimental error). However, the dependence of the nanostructure motion direction on the current direction is not trivial. In order to explain this dependence we have to take into consideration the atomic structure of the Si surface and its diffusion anisotropy. Several authors have found that on $\mathrm{Si}(100)$ surface diffusion is much faster along the dimer rows than perpendicularly to them. The ratio between the reported values of parallel and perpendicular diffusion coefficients ranges between 4 and 1000 [9, 18] depending on the temperature. In the following section we develop a model to understand the nanostructure motion under electromigration on anisotropic surfaces.

\section{Model of nanostructure motion under electro- migration}

In order to model the behavior of holes and islands under electromigration in $\mathrm{Si}(100)$, we use a basis of unit vectors $\vec{u}$ and $\vec{w}$ oriented along the [011] (we consider it equal to the direction of the dimer rows inside the nanostructure 


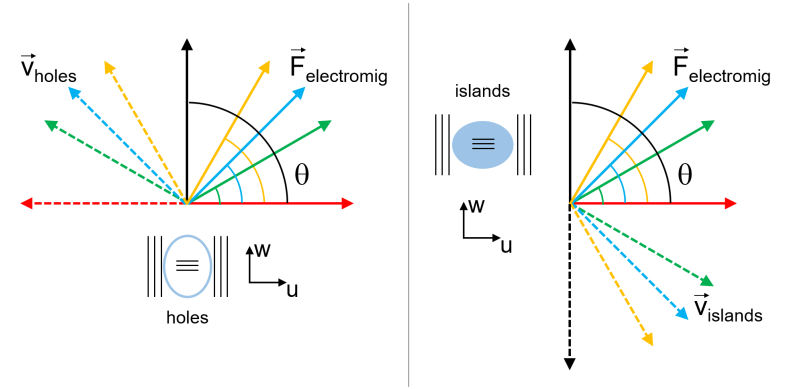

Figure 3: Graphical summary of the velocity direction of holes and islands under the effect of an electromigration force. Arrows with full lines represent directions of the force due to the electric field. They form an angle $\theta$ with $\vec{u}$ equal to $0^{\circ}, 30^{\circ}, 45^{\circ}, 60^{\circ}$, and $90^{\circ}$ for red, green, blue and yellow arrows respectively. The dashed arrows represent the velocity directions of holes (left) and islands (right) corresponding to force arrows with the same color.

for both islands and holes) and the [0-11] direction respectively, as shown in Figure 2. The force due to the electric field has a direction forming an angle $\theta$ with $\vec{u}$. We consider dimers as mobile species (see the KMC section) and discuss here the case of a one-atom deep hole. Analogous expressions can be derived for one-atom thick islands. As the experimentally measured velocity does not depend on the size of the nanostructure, terrace diffusion is considered to be the dominant mechanism of mass transport [17]. Atoms diffuse with a bias due to the sum of two forces: a wind force (momentum exchange between conducting electrons and adatoms) and a direct force (electrostatic force from the applied electric field on atoms). We assume that the prevailing force $\vec{F}$ is the direct force, in the same direction of the electric current, as also supposed in 9, 10 and usually found on semiconductor surfaces [19]. This force can be written $\vec{F}=Z^{*} \cdot e \cdot|\mathrm{E}| \cdot(\cos \theta \vec{u}+\sin \theta \vec{w})$, where $Z^{*}$ is the adatom effective valence, $e$ is the electron charge, and $|\mathrm{E}|$ is the magnitude of the applied electric field.

The surface mass fluxes (number of atoms per unit time, per unit length) at the exterior and at the interior of the hole depend on the concentration of diffusing species, on their diffusion coefficient, and on the force acting on them. These fluxes can be written as vectors $\vec{j}_{\text {ext }}$ and $\vec{j}_{\text {int }}$ with components $(\alpha=\mathrm{u}$ or $\mathrm{w})$ :

$$
j_{i, \alpha}=\frac{c}{k T} \sum_{\beta=u, w} F_{\alpha} D_{i, \alpha \beta}
$$

where if $i=i n t$ the diffusion tensor reads

$$
D_{i n t}=\left(\begin{array}{cc}
D_{u u}=D_{\|} & D_{u w}=0 \\
D_{w u}=0 & D_{w w}=D_{\perp}
\end{array}\right)
$$

whereas if $i=e x t$ the diffusion tensor reads

$$
D_{e x t}=\left(\begin{array}{cc}
D_{\perp} & 0 \\
0 & D_{\|}
\end{array}\right)
$$

$c$ is the dimer concentration, considered constant, $\mathrm{k}$ is the Boltzmann constant, $\mathrm{T}$ is the temperature. $D_{\perp}$ and

\begin{tabular}{c}
\hline$\vec{j}_{\text {ext }}=A \cdot\left(D_{\perp} \cdot \cos \theta \cdot \vec{u}+D_{\|} \cdot \sin \theta \cdot \vec{w}\right)$ \\
\hline$\vec{j}_{i n t}=A \cdot\left(D_{\|} \cdot \cos \theta \cdot \vec{u}+D_{\perp} \cdot \sin \theta \cdot \vec{w}\right)$ \\
\hline$\vec{v}_{h}=A \cdot a^{2}\left(D_{\|}-D_{\perp}\right) \cdot(-\cos \theta \cdot \vec{u}+\sin \theta \cdot \vec{w})$ \\
\hline$\vec{v}_{i s l}=A \cdot a^{2}\left(D_{\|}-D_{\perp}\right) \cdot(\cos \theta \cdot \vec{u}-\sin \theta \cdot \vec{w})$ \\
\hline
\end{tabular}

Table 1: Fluxes and velocities of holes and islands. $A=c \cdot \frac{Z^{*} \cdot e \cdot|\mathrm{E}|}{k T}$

$D_{\|}$are the surface diffusion coefficients respectively perpendicular and parallel to the dimer rows. There is no Fick-diffusion term proportional to the gradient of the concentration in the mass flux $\vec{j}$ because we assume that the system is in the diffusion-limited regime, where the concentration is fixed to its equilibrium value close to atomic steps. This assumption is valid as long as the attachmentdetachment dynamics at the steps is fast enough, a condition which should be observed in our high-temperature experiments where the kink density at steps is large.

If the interior and exterior fluxes $\vec{j}$ are not equal, a net mass flux at the edges of the hole results in an advancement velocity. In steady state regime the hole shape is fixed and the hole velocity is enforced by mass conservation:

$$
\vec{v}_{h}=a^{2} \cdot\left(\vec{j}_{\text {ext }}-\vec{j}_{\text {int }}\right)
$$

where $a$ is an atomic size. If instead of holes we consider one-atom thick islands, the growth direction of the steps is opposite to the previous case, therefore the direction of island motion is opposite to that derived for holes.

The analytical expressions of the $\vec{j}$ fluxes, the hole velocity $\vec{v}_{h}$ and the island velocity $\vec{v}_{i s l}$ that result from previous equations are reported in table 1 .

This model emphasizes the dependence between nanostructure (island or hole) motion and diffusion anisotropy, since for $D_{\|}=D_{\perp}, \vec{v}_{h}=\vec{v}_{i s l}=\overrightarrow{0}$. Furthermore these equations directly give the angle $\gamma$ formed by the velocity of the nanostructure with the dimer rows direction $\vec{u}$. For this purpose it is sufficient to compare the velocity expressions with $\vec{v}=|v| \cdot(\cos \gamma \cdot \vec{u}+\sin \gamma \cdot \vec{w})$ to extract $\gamma=180^{\circ}-\theta$ for $2 \mathrm{D}$ holes and $\gamma=-\theta$ for $2 \mathrm{D}$ islands.

The expression of the nanostructure velocity obtained with this model explains all the experimentally observed displacement direction of holes and islands. For instance it correctly predicts that when $\theta=45^{\circ}$ holes move perpendicularly to the electric field $\left(\gamma-\theta=90^{\circ}\right.$, see table 2$)$. Figure 3 schematically summarizes the motion directions of holes and islands according to the angle formed between the electric field and the dimer rows inside the nanostructure. Notice that the model reproduces the experimental observation that the absolute value of the velocity of the center of mass does not depend on the direction of the applied field. Moreover it does not depend on the nanostructure shapes, when isolated nanostructures are considered (that means nanostructures that do not interact with their local environment e.g. steps). The good agreement between the model and the experimental results suggests that the hypothesis of atoms drifting in the same direction of the electric current is correct. 


\section{Effective energy}

Figure 4 a shows the trajectories of several one-atom deep holes as a function of time. For all the nanostructures the distance from the initial position increases linearly with time (i.e. constant velocity). Only at the end of the motion their velocity decreases because they are disappearing as a consequence of Ostwald ripening (see later). From the above model (see table 1) we can develop the expression of the nanostructure velocity modulus:

$$
\begin{aligned}
\left|v_{h}\right|=\left|v_{i s}\right|=a^{2} \cdot c_{0} \cdot e^{\frac{-E_{c}}{k T}} \cdot \frac{Z^{*} \cdot e \cdot|\mathrm{E}|}{k T} \\
\cdot\left(D_{0, \|} \cdot e^{\frac{-E_{11}}{k T}}-D_{0, \perp} \cdot e^{\frac{-E_{\perp}}{k T}}\right)
\end{aligned}
$$

The dimer concentration $c$ depends on the temperature and on a dimer creation energy $E_{c}$. The diffusion coefficients are developed considering the prefactors $\left(D_{0, \|}\right.$ and $\left.D_{0, \perp}\right)$ and the diffusion energies $\left(E_{\|}\right.$and $\left.E_{\perp}\right)$. If $D_{\perp}$ is much lower than $D_{\|}$, then we can write:

$$
\left|v_{h, i s}\right| \propto \frac{\mathrm{E} \cdot e^{-\frac{E_{c}+E_{11}}{k T}}}{k T}=\frac{\mathrm{E} \cdot e^{-\frac{E_{\text {eff }}}{k T}}}{k T}
$$

Measuring the velocity at different temperatures, we estimate the effective energy $E_{\text {eff }}=E_{c}+E_{\|}$(see Figure 4). The slight difference between the data points obtained from holes and those obtained from islands is negligible. The data points obtained by Métois et al. [11] for island motion at $\mathrm{Si}$ vapor pressure equilibrium are also reported for comparison, they are in good agreement with our measurements. Notice that, if $E_{\perp}>>E_{\|}$, then $D_{\perp}$ can be neglected and the slope in Figure 4 corresponds to $E_{\text {eff }}$. Also if $E_{\perp} \approx E_{\|}$the slope is close to $E_{\|}$, but the intercept with the y axis significantly changes (therefore the pre-exponential cannot be related to physical constants).

A linear fit through the points gives an effective energy of $E_{\text {eff }}=2.0 \pm 0.1 \mathrm{eV}$. This value is close to that attributed by Keeffe et al.20 to the sum of the surface diffusion plus the creation energy of the diffusing species $(2.3 \mathrm{eV})$. A similar value is also reported for the effective energy obtained from another phenomenon involving $\mathrm{Si}(100)$, i.e. dewetting from a $\mathrm{SiO}_{2}$ substrate $(2 \mathrm{eV}[21])$. If we take the binding energy found at ambient temperature by Swartzentruber [22] for units at the end of dimer rows, $E_{c}=1.0 \pm 0.1$ $\mathrm{eV}$, equal to the dimer creation energy, then we obtain $E_{\|}=E_{\text {eff }}-E_{c}=1.0 \pm 0.2 \mathrm{eV}$. This value is equal to the

Table 2: Angles between the electric field or the hole motion direction with the [011] direction and corresponding figure. Also the angle between the current and the motion direction is reported $(\gamma-\theta)$.

\begin{tabular}{c|c|c|c}
\hline$\theta^{\circ}$ & $\gamma^{\circ}$ & $\gamma-\theta^{\circ}$ & experiment \\
\hline \hline 0 & 180 & 180 & Figure $1 \mathrm{~b}-\mathrm{c}$ \\
90 & 90 & 0 & Figure $1 \mathrm{~d}-\mathrm{e}$ \\
45 & 135 & 90 & Figure 1f-g \\
\hline
\end{tabular}

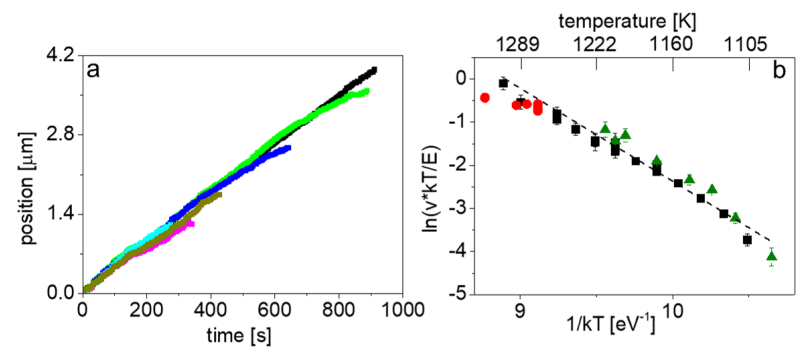

Figure 4: a: Position as a function of time of seven holes at $1150 \mathrm{~K}$. b: logarithm of the experimentally measured hole and island velocity multiplied by $\mathrm{kT}$ and divided by the electric field as a function of $1 /(\mathrm{kT})$. Black square and green triangles refer to our measurements of island and hole motion respectively. For comparison, we also report the data obtained by Metois et al. 11] as red circles. A linear fit through the data gives an effective activation energy of $E_{\text {eff }}=2.0 \pm 0.1$ $\mathrm{eV}$.

activation energy of about $1 \mathrm{eV}$ for dimer motion below $400 \mathrm{~K} 23$, and compares reasonably well to the surface diffusion energy found from island densities during Si deposition between 300 and $600 \mathrm{~K}(0.7 \mathrm{eV})$ [24].

We now evaluate the effective valence $Z^{*}$. $Z^{*}$ can be obtained from equation 2 , using the experimental values summarized in table 3. For the calculation, not only the diffusion energies, but also the prefactors must be known. Values for both $D_{\|}$and $D_{\perp}$ have been given by Doi et al. 25] who studied surface diffusion on $\mathrm{Si}(100)$ by measuring the size of denuded zones (distance between a step and the first island on the terrace) after Si deposition and annealing. Because of the experimental method they used, we rather suggest they actually found $D_{11} \cdot c$ and $D_{\perp} \cdot c$ (see table 3). We therefore find $7<Z^{*}<25$.

The effective charge we found is of the order of magnitude of that estimated by Stoyanov [8] for $\operatorname{Si}(100)\left(Z^{*}=1\right)$ and consistent with the results of Saul et al. 26 who found $Z^{*}>1.3$. Notice that, while we are confident on our velocity measurements, the effective charge value strongly depends on the order of magnitude of the diffusivity [25] $(D \cdot c)$ that we have used.

\section{Nanostructures size and shape}

As discussed by Hamers et al. [27, the nanostructure shapes on $\mathrm{Si}(100)$ are determined by the energy difference of $\mathrm{S}_{A}$ steps and $\mathrm{S}_{B}$ steps (see Figure 1 1 ). For instance Chadi 16 calculated 0.01 and $0.15 \mathrm{eV}$ for $\mathrm{S}_{A}$ and $\mathrm{S}_{B}$ steps respectively. Without electromigration, the equilibrium shape of one-atom thick nanostructures is an ellipse [28. With increasing temperature the aspect ratio (short axis/long axis) increases (see supplementary material S2), and the nanostructures becomes more rounded as also reported in 28 .

In the experiments, the holes and islands areas change because of a combination of Ostwald ripening [29] (due to atom exchange with the surrounding terraces) and evaporation, that takes place at high temperature. The behaviour of holes and islands is quite different. Indeed while 
Table 3: Values used to evaluate the effective charge.

\begin{tabular}{c|c|c|c|c}
\hline$|\mathrm{E}|[\mathrm{V} / \mathrm{nm}]$ & $\left|v_{h}\right|[\mathrm{nm} / \mathrm{s}]$ & $D_{\|} \cdot a^{2} \cdot c\left[s^{-1}\right]$ & $D_{\perp} \cdot a^{2} \cdot c\left[s^{-1}\right]$ & $\mathrm{T}[\mathrm{K}]$ \\
\hline \hline $4 \cdot 10^{-7}$ & 0.9 & $3.6 \cdot 10^{-10}$ & $0.7 \cdot 10^{-10}$ & 1089 \\
$4 \cdot 10^{-7}$ & 7 & $11.3 \cdot 10^{-10}$ & $4.4 \cdot 10^{-10}$ & 1173 \\
\hline
\end{tabular}

2D islands always shrink, $2 \mathrm{D}$ holes shrink when they move in the direction of the electric field (i.e. when $\theta$ is $90^{\circ}$ in Figure 2 but grow when they move opposite to the electric field $\left(\theta=0^{\circ}\right)$. The area change versus temperature is shown in Figures $5 \mathrm{a}$ and $5 \mathrm{p}$ for holes and islands respectively. Since experimental growth and shrink rates depend on the nanostructure size and on its close environment, we only show average values (for various sizes) of the area change. The difference between holes and islands is strongly associated to the anisotropy of surface diffusion. Indeed, since Ostwald ripening of a nanostructure is proportional to the diffusion rate on the terraces surrounding the nanostructure [29, it is more important when diffusion outside holes/islands is high (i.e. when the dimer rows outside the nanostructure are in the direction of the electric field, $\left.\theta=90^{\circ}\right)$. When diffusion outside the nanostructures is limited $\left(\theta=0^{\circ}\right)$, Ostwald ripening takes place at a lower rate so that evaporation dominates over ripening.

For islands, both Ostwald ripening and evaporation lead to shrinking (see Figure $5 \mathrm{p}$ ). However, as ripening is faster when diffusion on the surrounding terraces is faster $\left(\theta=90^{\circ}\right)$, islands with dimer rows perpendicular to the electric field shrink faster than those with dimer rows parallel to the field.

For holes, evaporation leads to growth and Ostwald ripening to shrinking. When diffusion outside the holes is hindered $\left(\theta=0^{\circ}\right)$, evaporation prevails and the holes grow (full squares in Figure 5a). When Ostwald ripening dominates $\left(\theta=0^{\circ}\right)$, the holes tend to disappear (empty squares in Figure 5a). However at high temperature the data are very scattered because the two phenomena are competing and the error on the measured area change is large.

\section{Kinetic Monte Carlo model}

We have developed a simple model which is able to reproduce our experimental results using a standard kinetic Monte Carlo method (KMC). The model is inspired from that of Ghosh and Ranganathan [30] and from the description of electromigration developed by Pierre-Louis and Einstein [17. In order to describe the (100) reconstructed surface formed by dimer rows we use a simple cubic solid-on-solid lattice with periodic boundary conditions. Every position of the lattice represents a column of diffusing units and has a certain height. Our LEEM analysis does not give information on the nature of the diffusing units on $\mathrm{Si}(100)$, which could be atoms, dimers or vacancies. Exchange processes between the surface and the bulk could also take place. Adatoms have been rarely observed by STM because either they diffuse very fast or because single adatoms are very unfavorable, as concluded by Huang and Allen 31] (because dimer formation reduces the system energy by $1 \mathrm{eV}$ ). Also Bartelt et al. [32, Tromp and Mankos [33] and Swartzentruber [34] suggest that the diffusing unit is a dimer. We thus consider the diffusing units to be dimers.

A jump of a diffusing unit depends on the neighbors of the unit and on the destination site. It takes place with probability proportional to

$$
\exp \left(\frac{-E_{b}-E_{d}-E_{m}}{k T}\right)
$$

where $\mathrm{k}$ is the Boltzmann constant, $\mathrm{T}$ is the temperature and the three different energies $E_{b}, E_{d}$ and $E_{m}$ are explained in the following paragraphs.

$(\bullet)$ The binding energy $E_{b}$ of the diffusing unit reads:

$$
E_{b}=E_{V}+n_{d r} \cdot E_{d r}+n_{p r} \cdot E_{p r}
$$

Within this expression the diffusing unit is bound (i) to the underlying surface with energy $E_{V}\left(E_{V}=E_{\|}=1 \mathrm{eV}\right.$ corresponds to the order of magnitude of the diffusion energy of isolated units along dimer rows [23); (ii) to up to two other dimers of the same dimer row, $\left(0 \leq n_{d r} \leq 2\right)$, with energy $E_{d r}$; and (iii) to up to two other dimers of parallel dimer rows, $0 \leq n_{p r} \leq 2$, with energy $E_{p r}$. More details on the binding energies and a schematics of the column positions in the model are reported in the supplementary material S3.

$(\bullet) E_{d}$ is an energy introduced to take into account diffusion perpendicular to dimer rows. It is added only if the arrival site of the diffusing unit is on top of a different underlying dimer row. In comparison with the analytical model, $E_{V}+E_{d}=E_{\perp}$. We have used $E_{d}=0.1 \mathrm{eV}$.

$(\bullet) E_{m}$ is the energy change due to the electric field. It depends on the arrival site of the diffusing unit and on the direction of the electromigration force (see schematics of supplementary material S3) according to the expression $E_{m}=|F| \cdot a \cdot \cos \left(\frac{\pi}{2} \cdot b-\delta\right)$. $a$ is a lattice length that we take equal to 1 in all directions, $|F|$ is the electromigration force acting on a jump of unit length, previously written as $Z^{*} \cdot e \cdot|\mathrm{E}|$, and $b$ is an integer between 1 and 4 defining the arrival site, in anticlockwise order $(b=1$ for a jump to the right, $\mathrm{b}=2$ for a jump to the top...); $\delta$ is the angle between the electromigration force and the $\mathrm{x}$ axis. The order of magnitude of the experimentally applied electric field $|E|$ is $10^{-7} \frac{V \text { olt }}{\text { lattice-length }}$. We have evaluated an effective valence $Z^{*}$ of the order of 10 elementary electron charges, therefore we should use a force lower than $10^{-6} \frac{\mathrm{eV}}{\text { lattice-length }}$. However that value is too low to observe any change due 

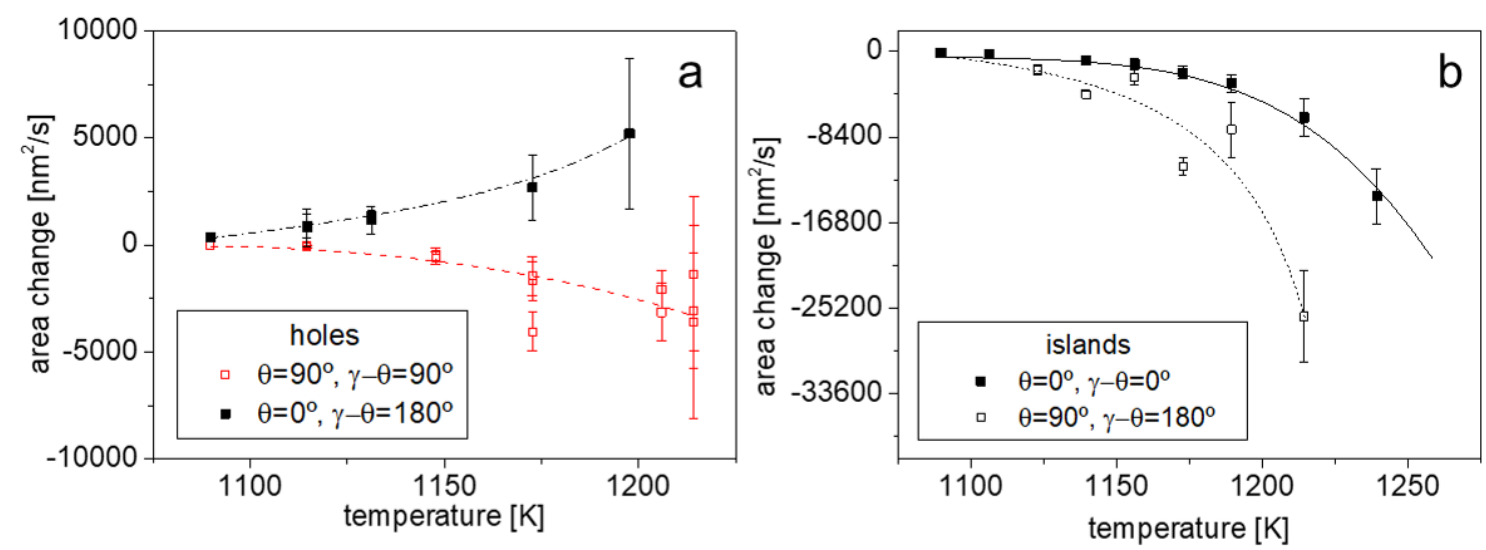

Figure 5: a:Average area change as a function of temperature for holes moving in the direction of the electric field (red empty squares) and for holes moving opposite to the electric field (black full squares). b:Average area decrease for islands moving in the direction of the electric field (full squares) and for islands moving opposite to the electric field (empty squares). Empty and full squares correspond to experimental results, while lines are a guide for the eyes. The error bars correspond to statistical errors that increase with temperature because of evaporation.
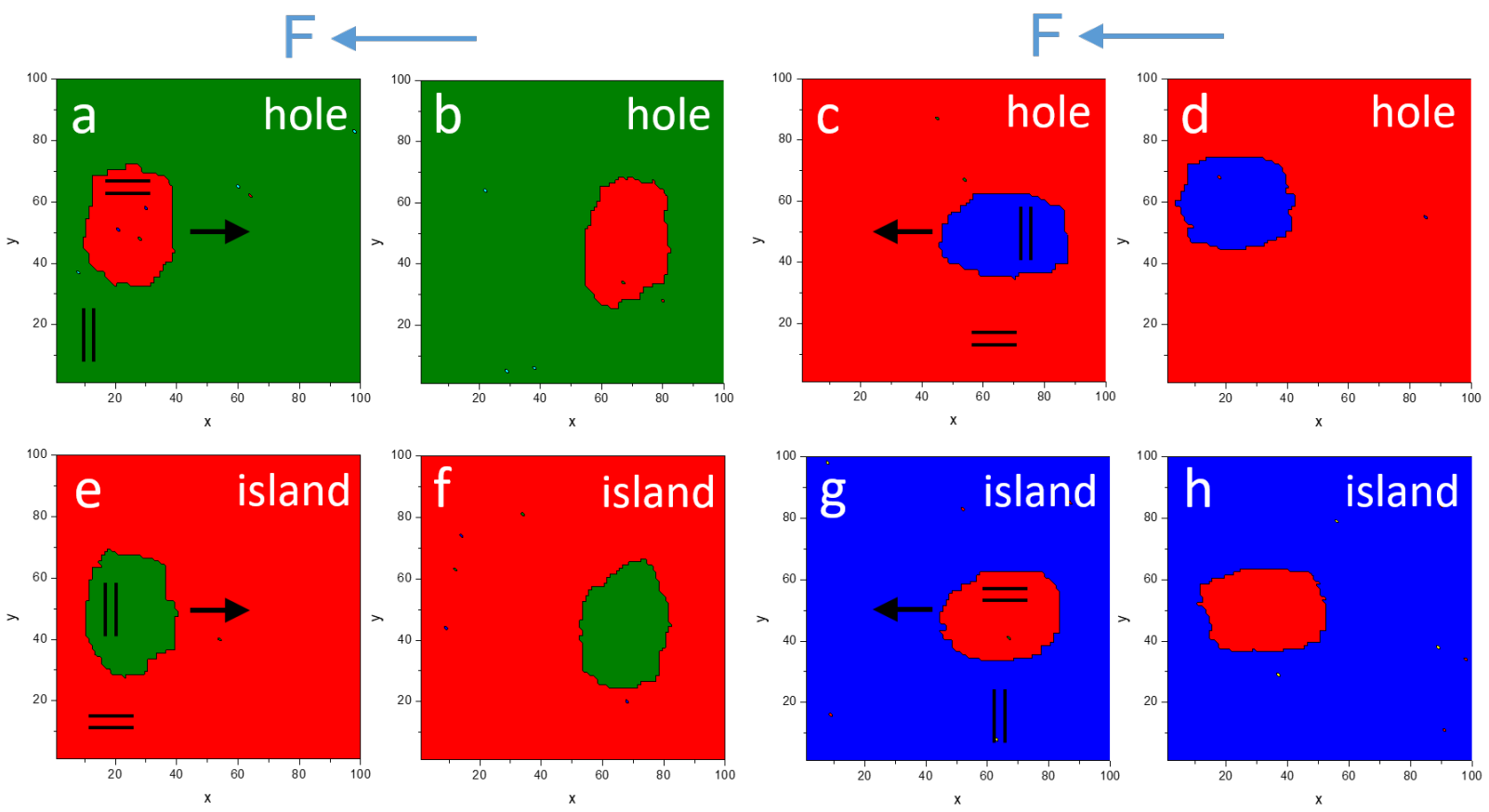

Figure 6: KMC snapshots of successive positions of nanostructures that move under the effect of a force to the left. The black lines represent the direction of the dimer rows in the images, the arrows show the motion directions. For this calculations kT $=0.05$; $(\mathrm{a}-\mathrm{b})$ : hole with horizontal dimer rows; (c-d): hole with vertical dimer rows; (e-f): island with vertical dimer rows; $(\mathrm{g}-\mathrm{h})$ : island with horizontal dimer rows. The height of the terraces decreases from green to red to blue. The green and blue regions have vertical dimer rows, while the red ones have horizontal dimer rows.

to electromigration in the simulations in reasonable time, therefore we have used $|F|=10^{-4} \frac{\mathrm{eV}}{\text { lattice-length }}$.

Many details of the (100) Si surface are neglected, such as the dimer buckling, the different possible diffusion sites (on top of dimers or in between dimer rows), or EhrlichSchwoebel barriers. We also consider the same effective charge for isolated dimers and dimers at steps. However the ingredients we use are sufficient to reproduce many features of the experimental behavior of islands and holes.

\section{Kinetic Monte Carlo simulations}

The shapes of the simulated nanostructures change as a result of the atom diffusion, but are approximately elliptical and their aspect ratio depends only on the values of the binding energies $E_{d r}$ and $E_{p r}$. Isotropic shapes are obtained when $E_{d r}=E_{p r}$. Simulated nanostructures or terraces have $\mathrm{S}_{A}$ steps flatter, with less kinks than $\mathrm{S}_{B}$ steps, as observed by Scanning Tunneling Microscopy (see for instance 23]). Figure 6 shows different types of holes and islands displacing in different directions under the 

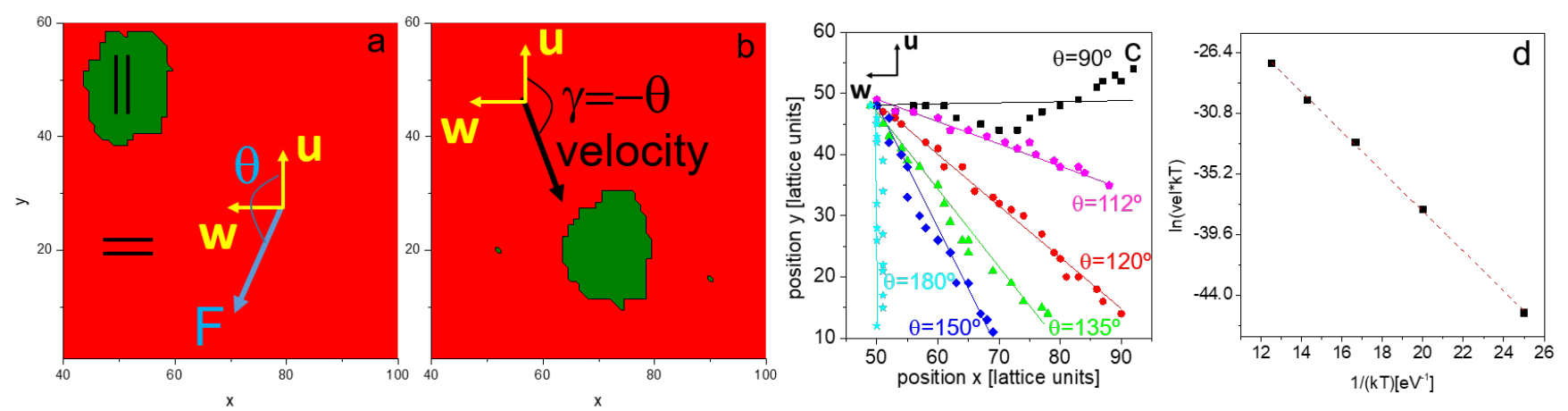

Figure 7: (a-b) KMC snapshots of two successive positions of an island that moves under the effect of a force at $\theta=150^{\circ}$. The black parallel lines show the direction of the dimer rows, the blue arrow show the direction of the electromigration force, forming an angle of $\theta$ with $\vec{u}$. c: trajectories of the center of mass of islands moving because of a force with angle $\theta$. Notice that the trajectories do not correspond to averages of several Monte Carlo simulations, so that the deviations from the straight lines depend on the pseudo-random numbers and have no physical meaning. d: logarithm of the simulated island velocity multiplied by $\mathrm{kT}$ as a function of $1 /(\mathrm{kT})$.

effect of the same electromigration force (directed from right to left). For instance Figure $6 \mathrm{a}$ and $6 \mathrm{p}$ show two snapshots of a hole that moves from left to right. All the obtained motion directions correspond to those experimentally observed and predicted by the analytical model. Under electromigration but without diffusion anisotropy $\left(E_{d}=0\right)$ holes and islands do not migrate in defined directions but only diffuse randomly. This confirms the previously obtained expression for the displacement of nanostructures, that depends on the difference of diffusion coefficients parallel and perpendicular to the dimer rows (equation 2].

Figure $7 \mathrm{a}$ and $7 \mathrm{p}$ show the displacement of an island when the force is directed at $\theta=150^{\circ}$. Figure 7 c shows the trajectories of islands for different orientations of the electromigration force. These simulations reproduce very well the experiments and the prediction of the analytical model.

The velocities obtained by simulating the motion of islands at different temperatures are plotted as a function of $1 /(\mathrm{kT})$ in Figure $7 \mathrm{~d}$, with a graph similar to that of Figure $4 \mathrm{~b}$. The slope of the linear fit provides an activation energy of $1.44 \pm 0.02 \mathrm{eV}$. This value corresponds to the sum of two energies used in the KMC model: (i) the diffusion energy in the fast direction $\left(E_{V}=E_{\|}=1 \mathrm{eV}\right)$ and (ii) the lowest energy to create adatoms $\left(E_{c}\right)$, that is the binding energy of a dimer in a kink position in the KMC model $\left(E_{p r}+E_{d r}=0.186+0.26 \mathrm{eV}\right)$. In order to obtain the experimental effective activation energy $(2 \mathrm{eV})$, the kink energy in the simulations must be $1 \mathrm{eV}\left(2-E_{V}=1\right)$. If we use $E_{p r}=0.42 \mathrm{eV}$ and $E_{d r}=0.58 \mathrm{eV}$, the effective activation energy found with the simulations is equal to the experimental one.

We have shown that our KMC model is able to reproduce the behaviors of hole and island motion. In order to test the predictive character of the model, we now use it for the analysis of more complex geometries in regimes where simple analytic predictions are not available.

\section{Edge instabilities induced by electromigration}

The instability of step edges appears as a natural candidate to challenge and test the predictive character of the KMC model. This instability is observed below 1100 $\mathrm{K}$ in both one-atom thick islands and holes. The shape of the holes and islands is then found to deviate from ellipsoids, and fingers form on the side where electromigration is down-step (Figure 8a). This effect is more visible on straight steps that develop crests and valleys whose amplitude increases with time. In the absence of electromigration, $\mathrm{S}_{A}$ steps are straight, whereas $\mathrm{S}_{B}$ steps are rough because of their different step stiffness [16. Under electromigration both types of steps are destabilized. The step on the right in Figure $8 \mathrm{a}$ (close to $\mathrm{a}_{B}$ step) develops crests pointing roughly horizontally, whereas the step on the left (close to a $\mathrm{S}_{A}$ step) has crests pointing at about $45^{\circ}$ with respect to the dimer row orientation (which are vertical on the bright terrace on the figure).

Such instabilities are reproduced by KMC simulations when both electromigration and anisotropic diffusion are implemented (Figure 8b). More precisely Figure 8p shows a terrace (red) with horizontal dimer rows one layer above another terrace (blue) with vertical dimer rows. At the beginning of the simulation (not shown) the steps of the red terrace are $S_{B}$ flat steps. Because of an electromigration force oriented from left to right, the terrace moves to the right and the advancing edge (the right one, where the current is in the step down direction) is destabilized. Without electromigration or without diffusion anisotropy $\left(E_{d}=0\right)$ there is no formation of crests and valleys (Figure 8 c shows the simulation of a terrace under electromigration but with isotropic diffusion, $\mathrm{E}_{d}=0$ ).

This instability does not correspond to that described by Bales and Zangwill [35, which originates in an increased flux from the lower side of an advancing step. Indeed, we rather have an increased flux of adatoms to the step from the upper terrace which should lead to step 

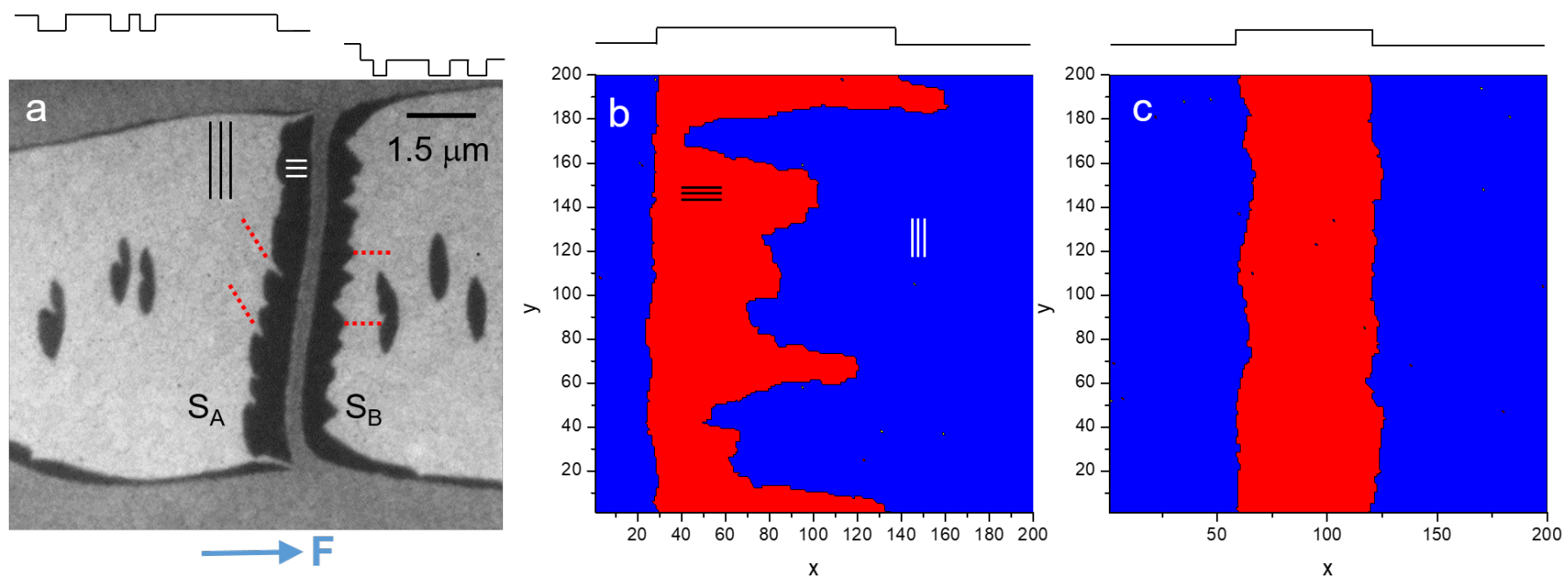

Figure 8: a: Black elongated holes with edges perturbed by electromigration. The grey regions are constituted by very small terraces. The black terrace at the left of the central grey region is lower than the bright terrace on the left. The step between them is some degrees away from a perfect $S_{A}$ step. The black terrace at the right of the grey central region is higher than the bright terrace on the right. The step between them is only some degrees away from a $\mathrm{S}_{B}$ step. The height profile is on the top of the image. b: KMC simulation of step instabilities. The red terrace has dimer rows oriented horizontally and is one monolayer higher than the blue one. The electromigration force is oriented from left to right and has a value of 0.01 , while all the other parameters have the values described in the text. The red terrace develops crests and valleys at the advancing edge. c: same conditions as in Figure a, but the diffusion is isotropic $\left(E_{d}=0\right)$; in this case the edges do not move and do not develop crests and valleys.

flattening in the Bales and Zangwill picture. Sato et al. [36] studied the linear stability of steps on $\mathrm{Si}(111)$ vicinal surfaces under electromigration with isotropic diffusion. They found an instability of step edges when the adatoms drift in the step down direction, and when the steps are not permeable. Step permeability is defined as the propensity of diffusing species to cross easily the steps. In our experiments and simulations, the instabilities also take place when the drift of dimers is in the step down direction. However, steps on $\mathrm{Si}(100)$ are known to be permeable [37, and the absence of instability observed in KMC simulations with isotropic diffusion also suggests that steps could be permeable in the KMC model. As a consequence, the prediction of Sato et al. does not correspond to our observations. In a different study, Sato et al. show that on $\mathrm{Si}(111)$ a down-step drift destabilizes the steps if there is a difference of diffusion coefficient between the lower and the upper terrace [38. The alternated anisotropy of terraces on $\mathrm{Si}(100)$ surfaces, which induces a difference in the mass fluxes on the two sides of the step, could play a role analogous to that played by the difference of diffusion coefficient. Further analytical modeling along the lines of ref [36, 38, would be needed to confirm the analogy. Nevertheless, the simple framework of our KMC simulations allowed us to reveal that diffusion anisotropy triggers a novel step instability caused by diffusion anisotropy that has not been identified in the previous experimental or theoretical literature. Our results call for further theoretical analysis and simulations to unravel the role of diffusion anisotropy on migration-induced step instabilities.

\section{Conclusions}

We have shown that under electromigration the diffusion anisotropy can affect the motion direction of nanostructures. This concept is demonstrated on $\mathrm{Si}(100)$ by insitu and real-time observations performed by LEEM of the motion of one-atom thick islands and holes under an electric field oriented along different directions. For a given $\theta$, angle between the electric field and the dimer rows inside the nanostructure, one-atom deep holes move at an angle of $180^{\circ}-\theta$ while one-atom thick islands move at angle equal to $-\theta$. The motion of nanostructures on $\operatorname{Si}(100)$ is possible thanks to the difference of diffusion coefficient parallel or perpendicular to dimer rows. From the velocity measurements of the motion of one-atom thick nanostructures we obtain an effective activation energy of $2.0 \pm 0.1 \mathrm{eV}$ given by the sum of a diffusion energy and a creation energy of the diffusing species. Electromigration at low temperatures when the electric field is mainly perpendicular to the $\mathrm{S}_{B}$ steps tends to de-stabilize $\mathrm{S}_{B}$ steps, leading to formation of crests and valleys. Finally, we have developed a Kinetic Monte Carlo model to simulate electromigration biased diffusion on $\operatorname{Si}(100)$. The simulations reproduce all the experimental observations and confirm the mechanism of motion proposed.

\section{Acknowledgments}

We acknowledge the financial support of the ANR grant HOLOLEEM (ANR-15-CE09-0012). We thank the CINaMPlanete facility and A. Ranguis for the technical support. We are grateful to A. Saul, B. Ranguelov and M. Michailov 
for stimulating discussions we had in the framework of the PHC Rila project (38663TB).

Declarations of interest: none

\section{References}

[1] C. Tao, W. G. Cullen, E. D. Williams, Visualizing the electron scattering force in nanostructures, Science 328 (2010) 736-740.

[2] I. Blech, E. Meieran, Electromigration in thin Al films, Journal of Applied Physics 40 (1969) 485.

[3] G. Ehrlich, F. G. Hudda, Atomic view of surface self diffusion: tungsten on tungsten, The Journal of Chemical Physics 44 (1966) 1039-1049.

[4] R. Schwoebel, Step motion on crystal surfaces. II, Journal of Applied Physics 40 (1969) 614-618.

[5] A. Latyshev, A. Krasilnikov, A. Aseev, S. Stenin, Effect of electric current on the ratio o the areas of the $(2 \times 1)$ and $(1 \times 2)$ domains at the clean (001) surface of silicon during sublimation, JETP Letters 48 (1989) 526-529.

[6] A. Latyshev, L. Litvin, A. Aseev, Peculiarities of step bunching on Si(001) surface induced by DC heating, Applied Surface Science 130 (1998) 139-145.

[7] S. Stoyanov, Heating current induced conversion between $2 \times 1$ and $1 \times 2$ domains at vicinal (001) Si surfaces - can it be explained by electromigration of Si atoms?, Japanese Journal of Applied Physics 29 (4) (1990) 659-662.

[8] S. Stoyanov, Electromigration induced step bunching on Si surfaces - how does it depend on the temperature and heating current direction, Japanese Journal of Applied Physics 30 (1) (1991) 1-6.

[9] M. Ichikawa, T. Doi, Study of Si(001) 2x1 domain conversion during direct current and radiative heatings, Applied Physics Letters 60 (1992) 1082.

[10] T. Doi, M. Ichikawa, S. Hosoki, K. Ninomiya, Anisotropic diffusion between the step-up and the step-down directions on a Si(001) surface, Physical Review B 53 (1996) 16609-16614.

[11] J.-J. Métois, J. C. Heyraud, A. Pimpinelli, Steady-state motion of silicon islands driven by a DC current, Surface Science 420 (1999) 250-258.

[12] K. Yagi, Reflection electron microscopy: studies of surface structures and surface dynamic processes, Surface Science Reports 17 (1993) 307-362.

[13] P. Müller, J.-J. Métois, Low distortion reflection electron microscopy for surface studies, Surface Science 599 (2005) 187195.

[14] D. Lee, J. Blakely, Formation and stability of large step-free areas on $\mathrm{Si}(001)$ and $\mathrm{Si}(111)$, Surface Science 445 (2000) 32-40.

[15] S. Ino, Some new techniques in Reflection High Energy Electron Diffraction (RHEED) application to surface structure studies, Japanese Journal of Applied Physics 16 (1977) 891-908.

[16] D. Chadi, Stabilities of Single-Layer and Bilayer Steps on Si(001) Surfaces, Physical Review Letters 59 (1987) 1691.

[17] O. Pierre-Louis, T. L. Einstein, Electromigration of single-layer clusters, Physical Review B 62 (20) (2000) 13697-13706.

[18] Y. Mo, M. Lagally, Anisotropy in surface migration of Si and Ge on Si(001), Surface Science 248 (1991) 313-320.

[19] H. Yasunaga, A. Natori, Electromigration on semiconductor surfaces, Surface Science Reports 15 (1992) 205-280.

[20] M. Keeffe, C. Umbach, J. Blakely, Surface self-diffusion on Si from the evolution of periodic atomic step arrays, Journal of Physics and Chemistry of Solids 55 (1994) 965-973.

[21] F. Cheynis, E. Bussmann, F. Leroy, T. Passanante, P. Müller, Dewetting dynamics of silicon-on-insulator thin films, Physical Review B 84 .

[22] B. Swartzentruber, Kinetics of Si monomer trapping at steps and islands on Si(001), Physical Review B 55 (1997) 1322-1325.

[23] B. Swartzentruber, M. Schacht, Kinetics of atomic-scale fluctuations of steps on $\mathrm{Si}(001)$ measured with variable-temperature STM, Surface Science 322 (1995) 83-89.
[24] Y. Mo, J. Kleiner, M. Webb, M. Lagally, Activation-energy for surface diffusion of Si on $\mathrm{Si}(001)$ - A Scanning-TunnelingMicroscopy study, Physical Review Letters 66 (1991) 19982001.

[25] T. Doi, M. Ichikawa, S. Hosoki, K. Ninomiya, Activation energies of Si adsorbate diffusion on a Si(001) surface, Surface Science 343 (1995) 24.

[26] A. Saúl, J.-J. Métois, A. Ranguis, Experimental evidence for an Ehrlich-Schwoebel effect on Si(111), Physical Review B 65 (2002) 075409.

[27] R. Hamers, U. Kohler, J. Demuth, Nucleation and growth of epitaxial silicon on $\mathrm{Si}(001)$ and $\mathrm{Si}(111)$ surfaces by scanning tunneling microscopy, Ultramicroscopy 31 (1989) 10-19.

[28] N. Bartelt, R. Tromp, E. Williams, Step capillary waves and equilibrium island shapes on $\mathrm{Si}(001)$, Physical Review Letters 73 (1994) 1656-1659.

[29] S. Curiotto, F. Cheynis, F. Leroy, P. Müller, Surface diffusion of $\mathrm{Au}$ on $\sqrt{3} \times \sqrt{3} \mathrm{Si}(111)$-Au studied by nucleation-rate and Ostwald-ripening analysis, Surface Science 647 (2016) L8-L11.

[30] P. Ghosh, M. Ranganathan, Submonolayer growth study using a solid-on-solid model for $2 \times 1$ reconstructed surfaces of diamondlike lattices, Surface Science 630 (2014) 174-181.

[31] Z. Huang, R. Allen, Diffusion of Si atoms and dimers on Si(100), Journal of Vacuum Science and Technology A 9 (1991) 876-879.

[32] N. Bartelt, W. Theis, R. Tromp, Ostwald ripening of twodimensional islands on $\mathrm{Si}(001)$, Physical Review B 54 (1996) 11741-11751.

[33] R. Tromp, M. Mankos, Thermal adatoms on Si(001), Physical Review Letters 81 (1998) 1050.

[34] B. Swartzentruber, Direct measurement of surface diffusion using atom-tracking Scanning Tunneling Microscopy, Physical Review Letters 78 (1996) 459-462.

[35] G. S. Bales, A. Zangwill, Morphological instability of a terrace edge during step-flow growth, Physical Review B 41 (1990) 5500-5508.

[36] M. Sato, M. Uwaha, Y. Saito, Y. Hirose, Step wandering induced by the drift of adatoms in a conserved system, Physical Review B 65 (2002) 245427.

[37] S. Tanaka, N. C. Bartelt, C. C. Umbach, R. M. Tromp, J. M. Blakely, Step permeability and the relaxation of biperiodic gratings on $\mathrm{Si}(001)$, Physical Review Letters 78 (1997) 3342.

[38] M. Sato, M. Uwaha, Y. Saito, Drift-induced step instabilities due to the gap in the diffusion coefficient, Journal of Crystal Growth 275 (2005) e129-e134. 